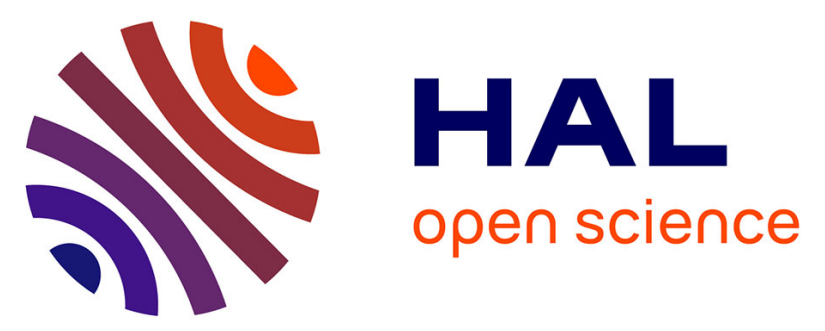

\title{
Les chirurgiens orthopédistes et traumatologues français sont-ils touchés par le syndrome de burnout? Résultats d'une enquête nationale
}

Grégoire Faivre, Guillaume Marillier, Jérémie Nallet, Sylvie Nezelof, Isabelle Clement, Laurent Obert

\section{To cite this version:}

Grégoire Faivre, Guillaume Marillier, Jérémie Nallet, Sylvie Nezelof, Isabelle Clement, et al.. Les chirurgiens orthopédistes et traumatologues français sont-ils touchés par le syndrome de burnout? Résultats d'une enquête nationale. Revue de Chirurgie Orthopédique et Traumatologique, 2019, 105, pp.234 - 239. 10.1016/j.rcot.2019.01.013 . hal-03486482

\section{HAL Id: hal-03486482 https://hal.science/hal-03486482}

Submitted on 20 Dec 2021

HAL is a multi-disciplinary open access archive for the deposit and dissemination of scientific research documents, whether they are published or not. The documents may come from teaching and research institutions in France or abroad, or from public or private research centers.
L'archive ouverte pluridisciplinaire HAL, est destinée au dépôt et à la diffusion de documents scientifiques de niveau recherche, publiés ou non, émanant des établissements d'enseignement et de recherche français ou étrangers, des laboratoires publics ou privés.

\section{(c) (1) $\$$}

Distributed under a Creative Commons Attribution - NonCommerciall 4.0 International 


\section{Mémoire original}

\section{Les chirurgiens orthopédistes et traumatologues français sont-ils touchés par le syndrome de burnout ? Résultats d'une enquête nationale.}

\section{Are French orthopedic and trauma surgeons affected by burnout? Results of a nationwide survey}

Grégoire Faivre ${ }^{a,}{ }^{,}$, Guillaume Marillier ${ }^{a}$, Jérémie Nallet ${ }^{a}$, Sylvie Nezelof ${ }^{b}$, Isabelle Clement ${ }^{c}$, Laurent Obert ${ }^{a}$

a Service de Chirurgie Orthopédique, Département Universitaire, $\mathrm{CHU}$ de Besançon, 3 Boulevard Alexandre Fleming, 25000 Besançon, France b Service de Psychiatrie de l'adulte, Département Universitaire, $\mathrm{CHU}$ de Besançon, 25000 Besançon, France

c Service de Médecine du travail, CHU de Besançon, 3 Boulevard Alexandre Fleming, 25000 Besançon, France

* auteur correspondant : Grégoire FAIVRE, Service de Chirurgie Orthopédique, Département universitaire, $\mathrm{CHU}$ de Besançon, 3 Boulevard Alexandre Fleming, 25000 Besançon,France.

Tel : 0684066888 
Mail : gr.faivre@gmail.com

Ne pas utiliser, pour citation, la référence française de cet article, mais celle de l'article original paru dans Orthopaedics \&Traumatology: Surgery \& Research, en utilisant le DOI ci-dessus.

\section{Résumé :}

Introduction :

Le syndrome de burnout est une pathologie qui touche les professions impliquées dans une relation d'aide. II associe un épuisement émotionnel (EE), une dépersonnalisation (DP), et une diminution de l'accomplissement personnel (DAP). La pratique de la chirurgie implique d'importantes responsabilités et est souvent associée à une charge de travail conséquente, ce qui expose les chirurgiens au risque de burnout. Cependant cette donnée n'est pas connue chez les chirurgiens orthopédistes et traumatologues français. Aussi nous avons mené une enquête prospective afin 1) d'évaluer la prévalence du syndrome de burnout chez les chirurgiens orthopédistes français 2) de tenter de déterminer les facteurs de risques et protecteurs.

\section{Hypothèse}

La prévalence du syndrome de burnout chez les chirurgiens orthopédiques français est au moins aussi importante que dans les autres spécialités médicales et chirurgicales.

\section{Matériels et méthodes}

Nous avons conduit une enquête nationale entre Février et Avril 2017, en diffusant un questionnaire numérisé par e-mail. Le syndrome de burnout était évalué par le score MBI (Maslach Burnout Inventory). Une évaluation des symptômes dépressifs était réalisée par le score GHQ-12 (General Health Questionnaire-12). Des données démographiques et sur la pratique professionnelle étaient également recueillies.

\section{Résultats}


Quatre cent quarante-et-un chirurgiens sur 1900 interrogés (23\%) ont répondu à l'enquête. L'âge moyen était de $50,2 \pm 10,1$ ans et on retrouvait $413(93,7 \%)$ hommes. Soixante-et-un (14\%) chirurgiens présentaient un degré élevé d’EE, 100 (23\%) un degré élevé de DP, et 82 (19\%) une DAP. Cent soixante-douze (39\%) chirurgiens présentaient des symptômes de burnout, représentés par un score pathologique à une des sous-échelles du MBI. Quarantesept (10\%) avaient un score pathologique dans 2 ou 3 des sous-échelles du MBI, correspondant à un syndrome de burnout sévère. Cent quatre-vingt-treize (43\%) participants ne recommandaient pas à leurs enfants de pratiquer la chirurgie orthopédique. Des idées suicidaires étaient déclarées par 38 (8\%) participants. Après analyse statistique, la pratique en secteur public (OR = 4,6; IC95 : 2,1-10,7; $p=0,0002$ ) et un score GHQ-12 pathologique $(O R=6,3 ; \operatorname{IC95}: 2,2-17,8 ; p=0,0006)$ étaient des facteurs de risque de burnout. La pratique d'activités extérieures (OR : 0,39; IC95:0,1-0,9; $p=0,0406$ ) et le sexe masculin (OR : 0,2;IC95:0,05-0,7; $p=0,0160$ ) étaient des facteurs protecteurs.

\section{Discussion}

Cette étude, malgré un taux de participation de 23\% ( $n=441 / 1900)$ permet d'avoir une idée du taux de burnout (39\% ( $n=172)$ présentant des symptômes de burnout, 10\% ( $n=47)$ de burnout sévère) des chirurgiens orthopédiques français. Le burnout est une pathologie qui a des conséquences sur la vie personnelle et professionnelle (augmentation des conduites suicidaires et du risque d'erreurs médicales et à une diminution de la qualité de vie des praticiens). Ces résultats rendent compte de l'importance de réaliser un dépistage et une prise en charge de cette pathologie chez les personnels soignants.

Type d'étude : niveau IV, étude prospective sans groupe contrôle, descriptive transversale. Mots clés : burnout, chirurgie orthopédique, suicide, Maslach Burnout Inventory 


\section{Introduction}

Au début des années 70, Freudenberger fut l'un des premiers à développer le concept de burnout [1], puis c'est Maslach [2], une psychiatre américaine, qui l'a défini comme un syndrome associant un épuisement émotionnel ( $E E)$, une diminution de l'accomplissement personnel (DAP), et une dépersonnalisation (DP) qui correspond au fait de considérer les patients comme des objets. Par définition le burnout se développe de manière chronique sur plusieurs années, et touche les professions impliquées dans une relation d'aide, comme celles du milieu médical et juridique. Le burnout est parfois associé à un véritable syndrome dépressif mais doit en être distingué sur plusieurs points : l'apparition chronique sans véritable facteurs déclenchants ou état psycho-pathologique antérieur et la limitation à la sphère professionnelle, du moins en début d'évolution.

Les conséquences de ce syndrome sont variées, et affectent la vie personnelle et professionnelle. Sur le plan personnel, le burnout a un impact négatif sur la vie de famille et est associé à une diminution de la qualité de vie [3]. Il est également lié aux pathologies cardio-vasculaire, au diabète de type 2 [4] et à un abus de substances addictives comme I'alcool ou le tabac. Les conduites suicidaires représentent la conséquence la plus grave du burnout [5]. Les patients sont les secondes victimes du burnout des praticiens, qui est associé à une augmentation des erreurs médicales [6] et à une diminution de la qualité des soins $[7,8]$.

Les professions médicales semblent être particulièrement exposées au risque de burnout [9], et notamment les spécialités chirurgicales [10]. Durant toute sa carrière, un chirurgien doit faire face à une multitude de situations pourvoyeuses de stress pour lui, mais également pour sa famille $[11,12]$. Aux Etats-Unis, le burnout semble toucher près d'un tiers 
des chirurgiens, avec des différences entre les spécialités. Les chirurgiens vasculaires, urologiques, et ORL figuraient parmi les plus touchés [10,13]. En France, des données sur le burnout des internes de chirurgie orthopédique ont déjà été publiées [14] mais aucune étude n'a encore étudié ce syndrome dans une population de chirurgiens orthopédistes seniors. Aussi nous avons mené une enquête prospective afin : 1) d'évaluer la prévalence du syndrome de burnout des chirurgiens orthopédistes et traumatologues français, 2) de tenter de déterminer les facteurs de risques et protecteurs. L'hypothèse était que la prévalence du syndrome de burnout chez les chirurgiens orthopédistes et traumatologues français est au moins aussi élevée que dans les autres spécialités médicales et chirurgicales.

\section{Matériels et méthodes}

\subsection{Population étudiée}

Nous avons conduit une enquête nationale, transversale qui s'adressait à l'ensemble des chirurgiens orthopédistes et traumatologues français (nombre investigué estimé à 1900). Le protocole de l'enquête avait été approuvé par la CNIL (Commission Nationale de I'Informatique et des Libertés) et le CPP (Comité de Protection des Personnes). Le recueil des données a été effectué à l’aide du logiciel LimeSurvey ${ }^{T M}$ (version 2.73.0+, application web).

\subsection{Recueil des données}

Un questionnaire a été diffusé par courriel à partir d'une liste d'adresses mail composée à l'aide des données du site de la SOFCOT (2222 adresses, dont 15\% erronées ou obsolètes laissant 1900 disponibles pour l'étude). Le recueil s'est déroulé entre Février et Avril 2017. Le mail contenait une invitation à participer à l'étude, une explication des modalités de celle$\mathrm{ci}$, ainsi qu'un lien hypertexte permettant de compléter le questionnaire en ligne. Une 
relance était réalisée au quinzième jour du recueil. La participation était basée sur le volontariat et les résultats étaient anonymes.

\subsection{Méthodes d'évaluation}

Le burnout était évalué à l'aide du Maslach Burnout Inventory (MBI) [2], un questionnaire validé de 22 questions, largement utilisé dans la littérature [15]. II explore à travers 3 souséchelles les 3 dimensions du burnout. Un score élevé dans une de ces sous-échelles était considéré comme un symptôme de burnout. Un score pathologique dans 2 ou 3 des souséchelles était fortement évocateur d'un syndrome de burnout sévère [2]. Les symptômes dépressifs étaient explorés par le score GHQ-12 [16,17], un questionnaire validé de 12 questions. Les valeurs des réponses étaient recodées selon une échelle bimodale, et un score supérieur ou égal à 4 évoquait une forte probabilité de détresse psychologique cliniquement significative. Des questions additionnelles étaient utilisées pour évaluer les caractéristiques démographiques, la satisfaction au travail, les données de la pratique professionnelle et les idées suicidaires.

\subsection{Méthodes statistiques}

Toutes les analyses statistiques ont été réalisées sous le logiciel SAS ${ }^{\text {TM }}$ (version 9.3, SAS Institute Inc., Cary, NC). Une analyse descriptive a initialement été effectuée, les variables quantitatives étant décrites par leur moyenne et leur écart-type, et les variables qualitatives par leurs effectifs, par modalité et leur pourcentage. Le questionnaire étant conçu de telle manière pour qu'il n'existe aucune donnée manquante pour le critère principal (MBI). L'ensemble des variables recueillies dans le questionnaire a été testé en univarié selon les scores d'épuisement, de dépersonnalisation et d'accomplissement du MBI. Une analyse multivariée a ensuite été réalisée selon un modèle de régression logistique afin d'étudier la 
relation entre les différentes dimensions du $\mathrm{MBI}$ (épuisement, dépersonnalisation et accomplissement) et les différentes variables recueillies dans le questionnaire. Les variables dont la p-value était inférieure à 0,2 de manière univariée ou présentant un intérêt clinique particulier, ont été introduites dans le modèle multivarié à l'exception des variables "sexe" et "âge" qui étaient des variables forcément étudiées dans le modèle multivarié. L'hypothèse d'écart à la linéarité était vérifiée pour chaque variable quantitative. Tous les tests avaient une erreur de type I bilatérale à 0,05.

\section{Résultats}

Quatre cent quarante-et-un chirurgiens orthopédistes et traumatologues français ont participé à l'étude sur les 1900 investigués (23\%). Les données démographiques et de la pratique professionnelle sont exposées dans le tableau 1. Afin de respecter l'anonymat des participants, nous n'avons pas de précisions supplémentaires à apporter par rapport au tableau 1, notamment sur l'origine géographique. On retrouvait $413(93,7 \%)$ hommes dans notre population et l'âge moyen était de $50,2 \pm 10,1$ ans avec 151 chirurgiens de plus de 55 ans (34\%) et 48 de moins de 35 ans (10,9\%). Deux cent trente (52\%) chirurgiens travaillaient dans le secteur privé. Les chirurgiens déclaraient en moyenne $56,9 \pm 12,8$ heures de travail

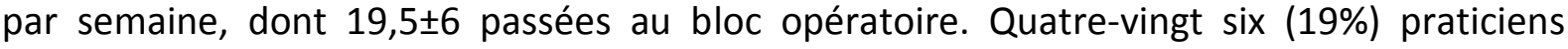
déclaraient travailler plus de 65 heures par semaine.

Concernant le syndrome de burnout (tableau 2), on retrouvait $14 \%(n=61) d^{\prime} E E, 23 \%(n=100)$ de DP, et $19 \%(n=82)$ de DAP. Cent soixante-douze (39\%) chirurgiens présentaient des symptômes de burnout, représentés par un score pathologique à une des sous-échelles du MBI. Quarante-sept (10\%) chirurgiens présentaient un score pathologique à 2 ou 3 des sous- 
échelles du MBI, évoquant un burnout sévère. On retrouvait $27 \%(n=7 / 26)$ de burnout sévère chez les jeunes orthopédistes (post-internat), 10\% ( $n=38 / 374)$ chez les praticiens hospitaliers et libéraux, et 4,8\% ( $n=2 / 41)$ chez les professeurs et maîtres de conférences des universités (PU-PH et MCU). De plus, 130 (29,5\%) chirurgiens présentaient des symptômes dépressifs selon le score GHQ-12. Les femmes semblaient présenter significativement plus de symptômes de burnout que les hommes $(p=0,0282)$ selon le score MBI (tableau 3). On ne retrouvait pas de différence significative pour le score $\mathrm{MBI}$ entre les praticiens du secteur privé $(n=230)$ et du secteur public $(n=211)$ (tableau 3$)$.

Après analyse statistique selon un modèle multivarié (tableau 4), la pratique en secteur public (OR = 4,6; IC95 : 2,1-10,7 ; P=0,0002) et les symptômes dépressifs (OR = 6,3 ; IC95\% : $2,2-17,8 ; p=0,0006)$ apparaissaient comme des facteurs de risque de burnout. Le sexe masculin (OR $=0,2 ;$ IC95: 0,05-0,76; $p=0,0160)$ et le fait de pratiquer des activités extérieures pour la gestion du stress (sports et loisirs en dehors du domicile ou du lieu de travail) $(O R=0,4 ; I C 95: 0,16-0,98 ; p=0,0406)$ ressortaient comme des facteurs protecteurs. La satisfaction au travail a été évaluée par plusieurs questions subjectives (tableau 5). Deux cent-quatre $(46 \%)$ chirurgiens interrogés déclaraient qu'ils recommanderaient à leurs enfants/proches de réaliser une carrière en chirurgie orthopédique. Deux cent vingt-neuf (51\%) d'entre eux déclaraient recommander à leurs enfants/proches de réaliser des études de médecine. Sur le plan financier, 194 (44\%) répondants se disaient « satisfait " ou " très satisfait » de leur salaire par rapport au travail fourni, et 174 (39\%) déclaraient envisager une retraite anticipée.

\section{Discussion}


En France, peu de travaux étudient le burnout dans la population médicale et à notre connaissance, aucun travail n'a été réalisé dans une population de chirurgiens orthopédistes et traumatologues français. Dans notre enquête, on retrouvait $14 \%$ d'EE $(n=61), 23 \%$ de DP $(n=100)$ et $19 \%(n=82)$ de DAP. Cent soixante-douze (39\%) chirurgiens présentaient des symptômes de burnout (score pathologique à une des sous-échelles du MBI) et 47 (10\%) présentaient des signes de burnout sévère (score pathologique à 2 ou 3 des sous-échelles du $\mathrm{MBI}$ ). De nombreux travaux ont étudié cette pathologie dans différentes spécialités médicales et chirurgicales (tableau 6). Dans notre effectif, les jeunes orthopédistes semblaient plus touchés par le burnout que leurs confrères PH ou PU-PH. Chez des jeunes chirurgiens orthopédistes américains, Sargent [19] retrouvait $28 \%$ d'EE, $24 \%$ de DP et $10 \%$ de DAP. Chez les internes français de chirurgie orthopédique [14], 40\% présentaient des signes de burnout sévère. Le jeune âge et le manque d'expérience semblent donc jouer un rôle dans l'apparition du burnout. Une des hypothèses est que l'acquisition d'expérience au cours d'une carrière chirurgicale permettrait de mieux gérer les diverses situations pourvoyeuses de stress.

Dans une vaste enquête sur près de 8000 chirurgiens américains toute spécialité confondue, Shanafelt et al. [6] rapportaient $31 \%$ d'EE, $26 \%$ de DP et $12 \%$ de DAP. Chez des chirurgiens vasculaires et colorectaux de Grande Bretagne, Sharma et al. [20] mettaient en évidence $31 \%$ d'EE, $21 \%$ de DP et $28 \%$ de DAP. Malgré un taux de burnout important retrouvé dans cette étude et dans la littérature, la chirurgie orthopédique semble être une des spécialités chirurgicales les moins touchées par ce phénomène comme le suggèrent Balch et al. [28]. Le burnout est aussi fortement présent dans les spécialités non chirurgicales, comme par exemple les médecins réanimateurs français, où Embriaco et al. [25] mettaient en évidence $19 \%$ d'EE, $37 \%$ de DP et $39 \%$ de DAP. 
Selon notre étude, le burnout est associé à la pratique en secteur public $(O R=4,6)$ et aux symptômes dépressifs $(O R=6,3)$. Concernant les symptômes dépressifs, il est impossible de conclure sur le fait qu'ils soient une conséquence ou une cause de burnout. Dans notre étude, les praticiens du secteur public étaient plus exposés au burnout que leurs confrères du privé, ce qui est l'inverse de ce qui retrouvé par Balch et al. [28] chez les chirurgiens américains. Cette différence entre chirurgiens français et américains est probablement liée aux modalités de pratique très différentes entre les deux pays. On retrouve notamment comme autre facteurs associés au burnout les poursuites judiciaires et le fait d'avoir un avenir incertain [21], ainsi qu'un temps de travail et un nombre d'astreintes et/ou gardes trop important $[22,24]$. Le sexe masculin était un facteur protecteur de burnout $(O R=0,2$; IC95 : 0,05-0,76 ( $p=0,0160))$ dans cette étude mais le nombre de femmes ayant participé à l'étude était peu important dans notre population $(n=28)$. Cependant, les femmes semblent semblent être plus exposées au burnout que leurs homologues masculins [3,23]. Ceci peut s'expliquer par le fait qu'elles subissent plus fortement les conflits entre vie privée et professionnelle (grossesse nécessitant un congé plus ou moins long, pression hiérarchique,...). Le fait d'avoir des enfants et d'avoir un bon équilibre entre vie personnelle et professionnelle sont d'autre facteurs protecteurs fréquemment retrouvés [3].

Cent quatre-vingt-treize (44\%) chirurgiens de notre étude déclaraient ne pas de recommander une carrière de chirurgien orthopédiste à leurs enfants ou proches. Des chiffres équivalents sont retrouvés aux Etats-Unis $[13,28]$. L'évolution la plus dramatique du syndrome de burnout est caractérisée par les conduites suicidaires. Dans cette étude, 38 (8,6\%) chirurgiens rapportaient des idées suicidaires au cours des 12 derniers mois, ce qui est 2 à 3 fois plus que la population générale française [29]. Ce taux est inférieur à celui retrouvé chez des chirurgiens italiens (17\%) et suédois (12\%) [5], ou chez les personnels des 
services de réanimation français (32\%) [25].

Ce travail présente plusieurs biais et limites. Premièrement, on retrouvait un faible taux de participation estimé à $23 \%(n=441 / 1900)$, ce qui est moins important que ce que l'on retrouve habituellement dans des enquêtes de ce type adressées à des médecins [30]. Cependant, cette faible participation est retrouvée dans de nombreuses autres études étudiant le burnout chez des médecins et chirurgiens [13,19,21-23]. De plus le mode de diffusion par email ne nous permettait pas de connaître exactement le nombre de chirurgiens ayant reçu et lu l'invitation, ce qui sous-estime probablement la participation. Secondairement, ce type d'étude introduit également un possible biais de réponse. En effet, il est difficile de savoir si des chirurgiens en burnout auront tendance à ne pas participer à ce type d'étude à cause de leur fatigue psychique, ou si au contraire ils seront plus enclins à fournir leur témoignage sur un sujet qui les concerne. Troisièmement, s'agissant d'une étude transversale, il nous est impossible de déterminer les liens de cause à effet entre les différents facteurs ainsi que leur direction. Enfin, il est important de rappeler que le score $\mathrm{MBI}$ est un score pronostique et ne signe en rien le diagnostic de burnout, ce qui doit rendre l'interprétation des résultats prudente. Ce travail présente également plusieurs forces. D’abord, à notre connaissance il s'agit de la seule étude du genre adressée à des chirurgiens orthopédistes et traumatologues français. Malgré un taux de participation pouvant sembler faible, la population étudiée regroupe plus de 400 individus, avec une répartition par genre (ratio $H / F: 93,7 \% / 6,3 \%)$ et un âge moyen $(50,2 \pm 10,1$ ans) semblable à ceux retrouvée dans I'atlas français de démographie médicale [31]. Ceci rend notre population comparable à celle des chirurgiens orthopédistes français. De plus, le questionnaire a été construit à partir d'outils validés comme le $\mathrm{MBI}$, largement utilisés dans la littérature, ce qui facilite la comparaison avec les autres études similaires. Enfin, en plus du burnout, cette enquête a 
recueilli de multiples données liées à la pratique professionnelle. Ceci nous a permis de mettre en évidence après analyse statistique multivariée, des facteurs associés au burnout.

\section{Conclusion}

Cette étude retrouve un taux significatif de burnout chez les chirurgiens orthopédistes et traumatologues français (10\% de burnout sévère, $40 \%$ présentant des symptômes de burnout). Ces résultats semblent équivalents à ceux retrouvés dans la littérature. Les conséquences de cette pathologie peuvent être dramatiques à la fois pour les médecins (conduites suicidaires...) et les patients (erreurs médicales...). Ces conclusions suggèrent que des mesures de prévention et de prise en charge doivent être entreprises au niveau local et national.

Conflits d'intérêts: Aucuns conflits d'intérêts à déclarer en relation avec ce travail. En dehors de ce travail LO déclare percevoir des honoraires de FX Solutions, Evolutis, et Springer. Les autres auteurs ne déclarent pas de conflits en dehors de ce travail.

Financement : Cette étude n’a reçu aucune subvention spécifique des secteurs public, privé ou non-lucratif.

Contribution des auteurs : Faivre Grégoire : (1) conception et mise en place de l'étude, acquisition, analyse et interprétation des données, (2) rédaction de l'article et révision critique pour contenu intellectuel important, (3) validation finale de la version soumise. Marillier Guillaume : (1) conception et mise en place de l'étude, acquisition, analyse et interprétation des données, (2) rédaction de l'article et révision critique pour contenu intellectuel important. Nallet Jérémie: (1) conception et mise en place de l'étude, 
acquisition, analyse et interprétation des données (4) Statistiques. Nezelof Sylvie : (1) conception et mise en place de l'étude, acquisition, analyse et interprétation des données, (2) rédaction de l'article et révision critique pour contenu intellectuel important. Clement Isabelle : (1) conception et mise en place de l'étude, acquisition, analyse et interprétation des données, (2) rédaction de l'article et révision critique pour contenu intellectuel important. Obert Laurent : (3) validation finale de la version soumise. 


\section{REFERENCES}

1. Freudenberger HJ. Staff Burn-Out. Journal of Social Issues. 1974;30:159- 165. doi:10.1111/j.1540-4560.1974.tb00706.x.

2. Maslach C, Jackson S, Letter M. Maslach Burnout Inventory Manual, 3rd edn. Palo Alto, CA: Consulting Psychologists Press, 1996.

3. Dyrbye LN, Shanafelt TD, Balch CM, Satele D, Sloan J, Freischlag J. Relationship between work-home conflicts and burnout among American surgeons : a comparison by sex. Arch Surg 1960;146:211-7.

4. Melamed S, Shirom A, Toker S, Berliner S, Shapira I. Burnout and risk of cardiovascular disease: evidence, possible causal paths, and promising research directions. Psychol Bull 2006;132:327-53.

5. Wall M, Schenck-Gustafsson K, Minucci D, Sendén MG, Løvseth LT, Fridner A. Suicidal ideation among surgeons in Italy and Sweden - a cross-sectional study. BMC Psychol 2014;2:53.

6. Shanafelt TD, Balch CM, Bechamps G, Russell T, Dyrbye L, Satele D, et al. Burnout and medical errors among American surgeons. Ann Surg 2010;251:995-1000.

7. Firth-Cozens J, Greenhalgh J. Doctors' perceptions of the links between stress and lowered clinical care. Soc Sci Med 1982;44:1017-22.

8. Shanafelt TD, Bradley KA, Wipf JE, Back AL. Burnout and self-reported patient care in an internal medicine residency program. Ann Intern Med 2002;136:358-67.

9. Shanafelt TD, Boone S, Tan L, Dyrbye LN, Sotile W, Satele D, et al. Burnout and satisfaction with work-life balance among US physicians relative to the general US population. Arch Intern Med 2012;172:1377-85.

10. Campbell DA, Sonnad SS, Eckhauser FE, Campbell KK, Greenfield L. Burnout among 
American surgeons. Surgery 2001;130:696-702-705.

11. Green A, Duthie HL, Young HL, Peters TJ. Stress in surgeons. Br J Surg 1990;77:1154-

8.

12. Kent GG, Johnson AG. Conflicting demands in surgical practice. Ann R Coll Surg Engl $1995 ; 77: 235-8$.

13. Shanafelt TD, Balch CM, Bechamps GJ, Russell T, Dyrbye L, Satele D, et al. Burnout and career satisfaction among American surgeons. Ann Surg 2009;250:463-71.

14. Faivre G, Kielwasser H, Bourgeois M, Panouilleres M, Loisel F, Obert L. Burnout Syndrome in Orthopaedic and Trauma Surgery Residents. A Nationwide Survey. Orthop Traumatol Surg Res 2018;104:1291-1295.

15. Pulcrano M, Evans SRT, Sosin M. Quality of life and burnout rates across surgical specialties: A systematic review. JAMA Surg 2016;151:970-8

16. Goldberg D. Williams P, A user's guide to the General Health Questionnaire. Berkshire, UK: NFER-Nelson Publishing: 1988.

17. Whitley TW, Allison EJ, Gallery ME, Cockington RA, Gaudry P, Heyworth J, et al. Workrelated stress and depression among practicing emergency physicians: an international study. Ann Emerg Med 1994;23:1068-71.

18. Streu R, Hansen J, Abrahamse P, Alderman AK. Professional burnout among US plastic surgeons: results of a national survey. Ann Plast Surg 2014;72:346-50.

19. Sargent MC, Sotile W, Sotile MO, Rubash H, Barrack RL. Quality of life during orthopaedic training and academic practice. Part 1: orthopaedic surgery residents and faculty. J Bone Joint Surg Am 2009;91:2395-405.

20. Sharma A, Sharp DM, Walker LG, Monson JRT. Stress and burnout in colorectal and vascular surgical consultants working in the UK National Health Service. Psychooncology 2008;17:570-6. 
21. McAbee JH, Ragel BT, McCartney S, Jones GM, Michael LM, DeCuypere M, et al. Factors associated with career satisfaction and burnout among US neurosurgeons: results of a nationwide survey. J Neurosurg 2015;123:161-73.

22. Kuerer HM, Eberlein TJ, Pollock RE, Huschka M, Baile WF, Morrow M, et al. Career satisfaction, practice patterns and burnout among surgical oncologists: report on the quality of life of members of the Society of Surgical Oncology. Ann Surg Oncol 2007;14:3043-53.

23. Rath KS, Huffman LB, Phillips GS, Carpenter KM, Fowler JM. Burnout and associated factors among members of the Society of Gynecologic Oncology. Am J Obstet Gynecol 2015;213:824.e1-9.

24. Kluger MT, Townend K, Laidlaw T. Job satisfaction, stress and burnout in Australian specialist anaesthetists. Anaesthesia 2003;58:339-45.

25. Embriaco N, Azoulay E, Barrau K, Kentish N, Pochard F, Loundou A, et al. High level of burnout in intensivists: prevalence and associated factors. Am J Respir Crit Care Med 2007;175:686-92.

26. Soler JK, Yaman H, Esteva M, Dobbs F, Asenova RS, Katic M, et al. Burnout in European family doctors: the EGPRN study. Fam Pract 2008;25:245-65.

27. Arigoni F, Bovier PA, Mermillod B, Waltz P, Sappino AP. Prevalence of burnout among Swiss cancer clinicians, paediatricians and general practitioners: who are most at risk? Support Care Cancer 2009;17:75-81.

28. Balch CM, Shanafelt TD, Sloan JA, Satele DV, Freischlag JA. Distress and career satisfaction among 14 surgical specialties, comparing academic and private practice settings. Ann Surg 2011;254:558-68.

29. Beck F. Tentatives de suicides et pensées suicidaires en France en 2010. http://inpes.santepubliquefrance.fr/Barometres/barometre-sante2010/pdf/BS2010_suicide.pdf - 05/2018 
30. Kellerman SE, Herold J. Physician response to surveys. A review of the literature. Am J Prev Med 2001;20:61-7.

31. Mourgues JM. Atlas de la démographie médicale en France. https://www.conseilnational.medecin.fr/sites/default/files/atlas_de_la_demographie_medicale_2017.pdf $05 / 2018$ 
Tableau 1 : données démographiques

Données démographiques et liées à la pratique professionnelle

\section{Sexe}

Ratio Homme/Femme

\section{Age}

$\leqq 35$ ans

Entre 36 et 45 ans

Entre 46 et 55 ans

Plus de 55 ans

Structure de travail

Secteur privé

Centre Hospitalier Universitaire

Hôpital périphérique / Centre hospitalier régional

Autre

Nombre d'heures de travail par semaine

$\leqq 45$

Entre 46 et 55

Entre 56 et 65

$>65$

Nombre d'astreintes / gardes par mois

$\leqq 4$

entre 5 et 8

entre 9 et 12

$>12$

Poursuites judiciaires durant les $\mathbf{5}$ dernières années

Oui

Non

Données manquantes n (441) $\quad \%$

$413 / 28 \quad 93,7 \% / 6,3 \%$

\begin{tabular}{cc}
\hline 48 & $10,9 \%$ \\
\hline 94 & $21,3 \%$ \\
\hline 148 & $33,6 \%$ \\
\hline 151 & $34,2 \%$ \\
\hline
\end{tabular}

230

$52.1 \%$

106

$24,0 \%$

76

$17,2 \%$

29

$6.6 \%$

$29 \quad 6.6 \%$

$\begin{array}{cc}86 & 19,5 \% \\ 134 & 30,4 \% \\ 135 & 30,6 \% \\ 86 & 19,5 \%\end{array}$

$\begin{array}{cc}160 & 36,3 \% \\ 159 & 36,1 \% \\ 72 & 16,3 \% \\ 50 & 11,3 \%\end{array}$

218

$49,4 \%$

213

$48,3 \%$

10

$2,3 \%$ 
Tableau 2 : résultats des scores Maslach Burnout Inventory (MBI) [2] et General Health Questionnaire-12 (GHQ-12) [16,17]

\begin{tabular}{|c|c|c|}
\hline Syndrome de burnout et troubles dépressifs & n (441) & $\%$ \\
\hline \multicolumn{3}{|l|}{ Score MBI : } \\
\hline \multicolumn{3}{|l|}{ Epuisement émotionnel } \\
\hline Elevé ( $\geq 30$ ) (pathologique) & 61 & $14 \%$ \\
\hline Modéré (entre 18 et 30) & 111 & $25 \%$ \\
\hline Bas $(\leq 17)$ & 268 & $61 \%$ \\
\hline \multicolumn{3}{|l|}{ Déshumanisation de la relation à l'autre } \\
\hline Elevé ( $\geq 12$ ) (pathologique) & 100 & $23 \%$ \\
\hline Modéré (entre 6 et 11) & 167 & $38 \%$ \\
\hline Bas $(\leq 5)$ & 174 & $39 \%$ \\
\hline \multicolumn{3}{|l|}{ Accomplissement personnel } \\
\hline Bas ( $\leq 33$ ) (pathologique) & 82 & $19 \%$ \\
\hline Modéré (entre 34 et 39) & 106 & $24 \%$ \\
\hline Elevé ( $\geq 40)$ & 253 & $57 \%$ \\
\hline Présentant au moins une sous-échelle pathologique & 172 & $38,9 \%$ \\
\hline Score pathologique à 1 sous-échelle & 125 & $28,3 \%$ \\
\hline Score pathologique à 2 sous-échelles & 39 & $8,8 \%$ \\
\hline Score pathologique à 3 sous-échelles & 8 & $1,8 \%$ \\
\hline \multicolumn{3}{|l|}{ Score GHQ-12 } \\
\hline$\geqq 12$ (pathologique) & 130 & $29,5 \%$ \\
\hline$<12$ & 311 & $70,5 \%$ \\
\hline
\end{tabular}

MBI : Maslach Burnout Inventory; GHQ-12 : General Health Questionnaire-12 
Tableau 3 : Résultats du score Maslach Burnout Inventory (MBI) [2] en fonction du sexe et du secteur d'activité (secteur privé vs secteur public)

\begin{tabular}{|c|c|c|c|}
\hline & $\begin{array}{l}\text { Secteur public } \\
(n=211)\end{array}$ & $\begin{array}{l}\text { Secteur privé } \\
(n=230)\end{array}$ & p \\
\hline $\begin{array}{l}\text { Score pathologique à } 1 \text { des sous- } \\
\text { échelles du MBI }\end{array}$ & 65 & 60 & 0,2911 \\
\hline \multirow{2}{*}{$\begin{array}{l}\text { Score pathologique à } 2 \text { ou } 3 \text { des } \\
\text { sous échelles du MBI }\end{array}$} & 27 & 20 & 0,1685 \\
\hline & Femmes $(n=28)$ & Hommes $(n=413)$ & \\
\hline $\begin{array}{l}\text { Score pathologique à } 1 \text { des sous- } \\
\text { échelles du MBI }\end{array}$ & 13 & 112 & 0,0282 \\
\hline $\begin{array}{l}\text { Score pathologique à } 2 \text { ou } 3 \text { des } \\
\text { sous échelles du MBI }\end{array}$ & 1 & 46 & 0,3413 \\
\hline
\end{tabular}

$\mathrm{MBI}$ : Maslach Burnout Inventory 
Tableau 4 : facteurs associés au burnout selon un modèle d'analyse multivarié

\begin{tabular}{|c|c|c|c|}
\hline $\begin{array}{l}\text { Facteurs associés au burnout après analyse } \\
\text { statistique multivariée }\end{array}$ & OR & IC95\% & p \\
\hline \multicolumn{4}{|l|}{ Facteurs de risques } \\
\hline GHQ12 pathologique & 6,0321 & $2,2275-17,8877$ & 0,0006 \\
\hline Pratique en secteur public & 4,6820 & $2,1086-10,7316$ & 0,0002 \\
\hline \multicolumn{4}{|l|}{ Facteurs protecteurs } \\
\hline $\begin{array}{l}\text { Pratique d'activités extérieures pour la gestion } \\
\text { du stress }\end{array}$ & 0,3984 & $0,1671-0,9855$ & 0,0406 \\
\hline Sexe masculin & 0,2003 & $0,0540-0,7624$ & 0,0160 \\
\hline
\end{tabular}

OR : Odds Ratio ; IC95 : Intervalle de confiance à 95\% : GHQ-12 : General Health Questionnaire-12 [16,17] 
Tableau 5 : Evaluation de la satisfaction au travail, des idées suicidaires et des situations de harcèlement au travail.

\begin{tabular}{lcc}
\hline Satisfaction au travail, idées suicidaires, harcèlement au travail & $\mathbf{n}(\mathbf{4 4 1})$ & $\mathbf{\%}$ \\
\hline $\begin{array}{l}\text { Recommande à ses enfants de pratiquer la chirurgie orthopédique } \\
\text { Oui }\end{array}$ & 204 & $46,3 \%$ \\
\hline Non & 193 & $43,8 \%$ \\
\hline Ne se prononce pas & 44 & $10,0 \%$ \\
\hline Satisfaction du salaire par rapport au travail fourni & & \\
\hline Très insatisfait & 46 & $10,4 \%$ \\
\hline Insatisfait & 101 & $22,9 \%$ \\
\hline Ni satisfait ni insatisfait & 100 & $22,7 \%$ \\
\hline Satisfait & 149 & $33,8 \%$ \\
\hline Très satisfait & 45 & $10,2 \%$ \\
\hline Retraite anticipée envisagée & & \\
\hline Oui & 174 & $39,5 \%$ \\
\hline Non & 244 & $55,3 \%$ \\
\hline Ne se prononce pas & 23 & $5,2 \%$ \\
\hline Idées suicidaires durant les 12 derniers mois & & \\
\hline Oui & & \\
\hline Non & & \\
\hline Ne se prononce pas & 38 & $8,6 \%$ \\
\hline Situations de harcèlement au travail durant la dernière année & 398 & $90,2 \%$ \\
\hline Oui & 5 & $1,1 \%$ \\
\hline Non & 341 & $77,3 \%$ \\
\hline Ne se prononce pas & 23 & $5,2 \%$ \\
\hline
\end{tabular}


Tableau 6 : Comparaison des taux de burnout selon les spécialités médicales et chirurgicales, parmi des populations de praticiens seniors.

\begin{tabular}{|c|c|c|c|c|c|c|}
\hline Auteurs & $\begin{array}{l}\text { Spécialités étudiées, pays } \\
\text { d'étude }\end{array}$ & $\begin{array}{l}\text { Nombre de } \\
\text { participants }\end{array}$ & $\begin{array}{l}\text { Taux de } \\
\text { participation (\%) }\end{array}$ & $\begin{array}{l}\mathrm{EE} \\
(\%)\end{array}$ & DP (\%) & $\begin{array}{l}\text { DAP } \\
\text { (\%) }\end{array}$ \\
\hline Notre étude & $\begin{array}{l}\text { Chirurgie orthopédique, } \\
\text { France }\end{array}$ & 441 & 22 & 14 & 23 & 19 \\
\hline \multicolumn{7}{|l|}{$\begin{array}{l}\text { Spécialités } \\
\text { chirurgicales }\end{array}$} \\
\hline $\begin{array}{l}\text { Streu et al. } \\
{[18]}\end{array}$ & Chirurgie plastique, USA & 506 & 71 & 29 & 16 & 5 \\
\hline $\begin{array}{l}\text { Sargent et al. } \\
{[19]}\end{array}$ & $\begin{array}{l}\text { Jeunes chirurgiens } \\
\text { orthopédiques, USA }\end{array}$ & 264 & 24 & 28,4 & 24,8 & 10 \\
\hline $\begin{array}{l}\text { Shanafelt et } \\
\text { al. [13] }\end{array}$ & $\begin{array}{l}\text { Toute spécialité chirurgicale } \\
\text { confondue, USA }\end{array}$ & 7905 & 32 & 31 & 26 & 12,8 \\
\hline $\begin{array}{l}\text { Sharma et al. } \\
{[20]}\end{array}$ & $\begin{array}{l}\text { Chirurgie vasculaire et } \\
\text { colorectale. Grande } \\
\text { Bretagne }\end{array}$ & 501 & 58 & 31,7 & 21,2 & 28,8 \\
\hline $\begin{array}{l}\text { McAbee et al. } \\
{[21]}\end{array}$ & Neurochirurgie, USA & 750 & 24 & 35,1 & 31,3 & 28 \\
\hline $\begin{array}{l}\text { Kuerer et al. } \\
\text { [22] }\end{array}$ & Chirurgie oncologique, USA & 549 & 36 & 24,1 & 15,2 & 9,6 \\
\hline $\begin{array}{l}\text { Rath et al. } \\
\text { [23] }\end{array}$ & Gynécologie, USA & 369 & 34 & 30 & 10 & 11 \\
\hline \multicolumn{7}{|l|}{$\begin{array}{l}\text { Spécialités } \\
\text { médicales }\end{array}$} \\
\hline $\begin{array}{l}\text { Kluger et al. } \\
{[24]}\end{array}$ & Anesthésie, Australie & 422 & 60 & 20 & 20 & 36 \\
\hline $\begin{array}{l}\text { Embriaco et } \\
\text { al. [25] }\end{array}$ & Médecine intensive, France & 978 & 82,5 & 19 & 37 & 39 \\
\hline $\begin{array}{l}\text { Soler et al. } \\
{[26]}\end{array}$ & $\begin{array}{l}\text { Médecine générale, Europe } \\
\text { (12 pays) }\end{array}$ & 1393 & 41 & 43 & 35 & 32 \\
\hline $\begin{array}{l}\text { Arigoni et al. } \\
\text { [27] }\end{array}$ & $\begin{array}{l}\text { Pédiatrie, oncologie, } \\
\text { médecine générale, Suisse }\end{array}$ & 371 & 66 & 33 & 22 & 20 \\
\hline
\end{tabular}

EE : épuisement émotionnel, DP : dépersonnalisation, DAP : diminution de l'accomplissement personnel. 